\title{
Duality of Ring and Ladder Diagrams and Its Importance for Many-Electron Perturbation Theories
}

\author{
Andreas Irmler, Alejandro Gallo, Felix Hummel, and Andreas Grüneis* \\ Institute for Theoretical Physics, TU Wien, Wiedner Hauptstraße 8-10/136, 1040 Vienna, Austria
}

(Received 14 March 2019; published 8 October 2019)

\begin{abstract}
We present a diagrammatic decomposition of the transition pair correlation function for the uniform electron gas. We demonstrate explicitly that ring and ladder diagrams are dual counterparts that capture significant long- and short-ranged interelectronic correlation effects, respectively. Our findings help to guide the further development of approximate many-electron theories and reveal that the contribution of the ladder diagrams to the electronic correlation energy can be approximated in an effective manner using second-order perturbation theory. We employ the latter approximation to reduce the computational cost of coupled cluster theory calculations for insulators and semiconductors by 2 orders of magnitude without compromising accuracy.
\end{abstract}

DOI: 10.1103/PhysRevLett.123.156401

Introduction.-The ongoing advancement of modern electronic structure theories over the past few decades led to a proliferation of computational studies on atoms, molecules, and condensed matter systems based on first principles. Electronic structure methods allow us to simulate a wide range of physically as well as chemically relevant material properties including thermodynamic stability, electric conductivity, magnetic ordering, and optical properties. Yet many of the most successful methods such as approximate density functional theory or many-electron perturbation theories rely partly on fortuitous error cancellation with farreaching consequences. Attempting to improve upon individual terms in the many-body perturbation expansion of the electronic exchange and correlation energy by employing higher levels of theory often has a contrary effect and deteriorates the achieved level of accuracy, making it hard to approach the exact result in a systematic manner. A profound physical understanding of the individual perturbation theory contributions to the ground and excited state energies of many-electron systems and their balance is, however, needed to develop novel (hybrid) methods that can complement existing methodologies to study systems with higher accuracy where needed. Here, we present a new method that makes it possible to decompose the many-body contributions to the correlation energies of $a b$ initio systems as well as the uniform electron gas in a manner that enables a deeper understanding of widely used approximations. Furthermore,

Published by the American Physical Society under the terms of the Creative Commons Attribution 4.0 International license. Further distribution of this work must maintain attribution to the author(s) and the published article's title, journal citation, and DOI. we demonstrate that the obtained findings can be used to improve the efficiency of many-electron theory calculations.

In many-body physics and quantum chemistry, approximate methods are often tailored to become exact in limiting cases. Examples for such electronic structure theories include (i) the random-phase approximation (RPA), which captures the leading order contributions to the correlation energy of the uniform electron gas in the high-density limit [1-3], (ii) the ladder theory, which works reliably in the low-density limit [4-6], and (iii) coupled cluster singles and doubles theory (CCSD) that combines the latter two approaches and is exact for two-electron systems [7,8]. Yet all of the methods mentioned above exhibit certain shortcomings for real materials, respectively, systematic overcorrelation, divergence of the correlation energy for metals, and a poor trade-off between computational cost and accuracy. In a remarkable manner, however, errors like the overcorrelation of the RPA cancel out when considering energy differences, and sometimes even mimic more sophisticated electronic correlation effects [9-11]. Furthermore, disregarding exchangelike interactions between electron pairs in coupled cluster theory systematically improves upon the achieved level of accuracy [12]. These examples illustrate that it is important to fully understand the effect of individual contributions as a function of the electronic density and interelectronic distance in order to guide the further development of more accurate and efficient approximations to the electronic correlation energy in strongly as well as weakly correlated systems.

Theory.-In this work we introduce a decomposition of the electronic transition pair correlation function and the corresponding correlation energy contributions. For the sake of brevity we will restrict the following discussion to the uniform electron gas (UEG) and turn to ab initio 
systems afterwards. The correlation energy can be written as an integral of the Coulomb potential $v\left(\mathbf{r}_{12}\right)=1 /\left|\mathbf{r}_{12}\right|$ and a function $g\left(\mathbf{r}_{12}\right)$ of the interelectronic radius $\mathbf{r}_{12}$ :

$$
E_{c}=\int d \mathbf{r}_{12} g\left(\mathbf{r}_{12}\right) v\left(\mathbf{r}_{12}\right)
$$

The function $g\left(\mathbf{r}_{12}\right)$ is called the transition pair correlation function. It can be explicitly given by

$$
g\left(\mathbf{r}_{12}\right)=\frac{1}{(2 \pi)^{3}} \sum_{a b i j} e^{i\left(\mathbf{k}_{i}-\mathbf{k}_{a}\right) \cdot \mathbf{r}_{12}}\left(2 t_{i j}^{a b}-t_{j i}^{a b}\right)
$$

and corresponds to the Fourier transform of the transition structure factor $S(\mathbf{q})$, recently studied in Refs. [13,14]. The indices $i, j$ and $a, b$ label occupied and virtual spatial orbitals, respectively. In the UEG, orbitals correspond to plane waves with respective wave vectors $\mathbf{k}_{i}, \mathbf{k}_{j}$ and $\mathbf{k}_{a}, \mathbf{k}_{b}$. The amplitudes $t_{i j}^{a b}$ are obtained by solving the underlying amplitude equations on the respective level of many-electron theory, such as second-order perturbation theory [15], coupled cluster singles and doubles theory $[7,8]$, the random-phase approximation [1-3], or ladder theory [5,6]. In coupled cluster doubles theory, the $t_{i j}^{a b}$ 's are obtained by solving the recursive amplitude equations $[16,17]$ that include all terms also present in the ring and the ladder approximation:

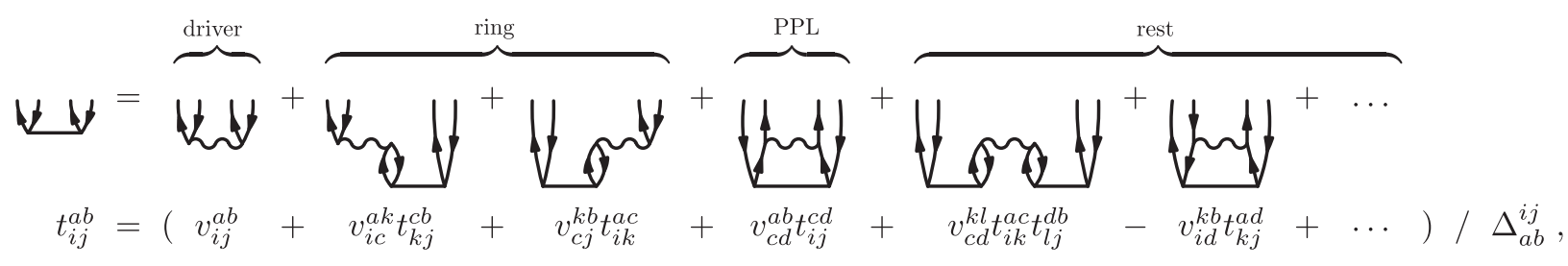

where $\Delta_{a b}^{i j}=\varepsilon_{i}+\varepsilon_{j}-\varepsilon_{a}-\varepsilon_{b}$ are the one-electron energy differences in the Hartree-Fock approximation. The amplitude equation above illustrates that coupled cluster methods perform a resummation of certain diagrams including particle-hole ring and particle-particle ladder (PPL) diagrams to infinite order coupling different diagrammatic channels. We note that single particle-hole excitation amplitudes $\left(t_{i}^{a}\right)$ make no contribution to the wave function and correlation energy of the uniform electron gas [18]. Given the amplitudes $t_{i j}^{a b}$ that solve Eq. (3) we can replace $t_{i j}^{a b}$ in Eq. (2) by the right-hand side of Eq. (3) to arrive at a decomposition of the transition pair correlation function into corresponding diagrammatic contributions that we refer to as driver, ring, PPL, and rest:

$$
\begin{aligned}
g^{\operatorname{CCSD}}\left(\mathbf{r}_{12}\right)= & g^{\text {driver }}\left(\mathbf{r}_{12}\right)+g^{\text {ring }}\left(\mathbf{r}_{12}\right)+g^{\mathrm{PPL}}\left(\mathbf{r}_{12}\right) \\
& +g^{\text {rest }}\left(\mathbf{r}_{12}\right) .
\end{aligned}
$$

Analogously, the transition structure factor $S^{\mathrm{CCSD}}(\mathbf{q})$ can be decomposed into its diagrammatic contributions. We note that a similar labeling of terms in the amplitude equations was employed in Refs. [18-22]. In contrast to previous work, we perform a decomposition only in one order of perturbation of the otherwise fully coupled amplitudes.

Results.-In the following we will discuss numerical results of the decomposed pair correlation function obtained for the UEG. The results shown in Fig. 1(a) have been computed employing a 54 electron gas simulation cell at a density corresponding to $r_{s}=5 a_{0}$ (atomic units) identical to the system used in Ref. [23]. The amplitudes are expanded in an orbital basis composed of 1863 plane waves. We first discuss $g^{\text {driver }}\left(\mathbf{r}_{12}\right)$, which captures the second-order correlation energy contribution to Eq. (1) and exhibits a minimum at $\mathbf{r}_{12}=\mathbf{0}$. Because of the employed finite one-electron basis set approximation, $\left.g^{\text {driver }}\left(\mathbf{r}_{12}\right)\right|_{\mathbf{r}_{12}=\mathbf{0}}$ lacks a derivative discontinuity as required by the cusp condition [24]. In passing we note that $g^{\text {driver }}\left(\mathbf{r}_{12}\right)$ of a single electron pair can be well approximated in the complete basis set (CBS) limit at short interelectronic distances using a Slater-type correlation function, $-\gamma^{-1} e^{-\gamma\left|\mathbf{r}_{12}\right|}$, where $\gamma$ is a parameter that increases with increasing density [23]. In decreasing order of absolute magnitude, the largest contributions to $g^{\mathrm{CCSD}}(\mathbf{0})$ originate from $g^{\text {driver }}\left(\mathbf{r}_{12}\right), g^{\text {rest }}\left(\mathbf{r}_{12}\right), g^{\mathrm{PPL}}\left(\mathbf{r}_{12}\right)$, and $g^{\text {ring }}\left(\mathbf{r}_{12}\right)$. In contrast to $\mathbf{r}_{12}=\mathbf{0}$, we find that the relative contribution of $g^{\text {ring }}\left(\mathbf{r}_{12}\right)$ to $g^{\mathrm{CCSD}}\left(\mathbf{r}_{12}\right)$ increases with increasing interelectronic distance, whereas $g^{\mathrm{PPL}}\left(\mathbf{r}_{12}\right)$ decays to zero rapidly. This observation demonstrates that the employed decomposition allows for an analysis of the interelectronic correlation strength and its dependence on the distance for individual diagrammatic contributions. We also note that the importance of ring diagrams for the correlation energy at large interelectronic distances is known and of particular significance to the correct description of dispersion interactions in semiconductors using the RPA $[10,11]$.

We now discuss the convergence of the decomposed pair correlation function with respect to the employed orbital basis set. $g^{\text {driver }}\left(\mathbf{r}_{12}\right)$ converges slowly to the complete basis set limit. This can be seen from $\Delta g^{\text {driver }}\left(\mathbf{r}_{12}\right)$ as depicted in 

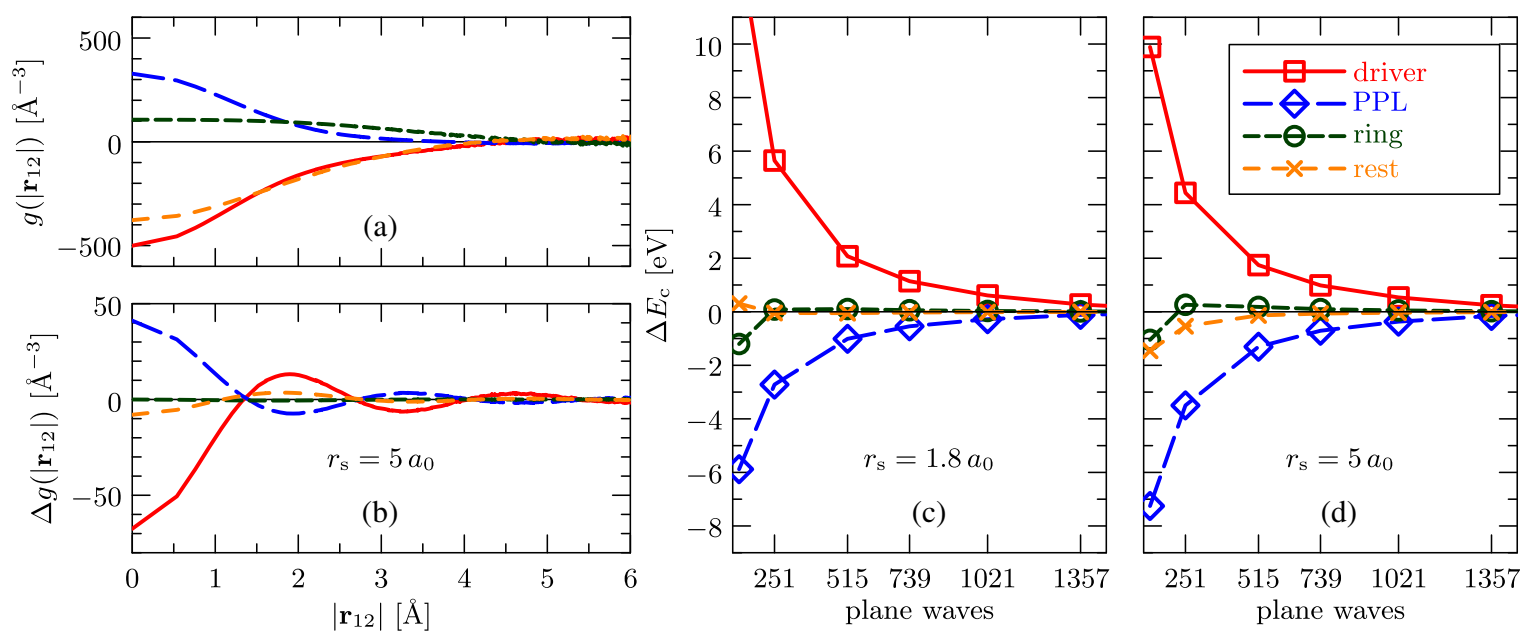

FIG. 1. (a) Pair correlation function contributions according to Eq. (4) using 1863 plane waves at $r_{s}=5 a_{0}$. (b) Difference between pair correlation function contributions obtained using 1863 plane waves and 1021 plane waves. (c),(d) CBS limit error of corresponding correlation energy contributions retrieved as a function of the plane wave basis set size for densities corresponding to $r_{s}=1.8 a_{0}$ and $r_{s}=5 a_{0}$, respectively.

Fig. 1(b), which corresponds to the difference of $g^{\text {driver }}\left(\mathbf{r}_{12}\right)$ calculated using 1021 and 1863 plane wave orbitals. This analysis demonstrates that the PPL term converges at a similar rate albeit with an opposite sign as indicated by $\Delta g^{\text {driver }}\left(\mathbf{r}_{12}\right)$ and $\Delta g^{\mathrm{PPL}}\left(\mathbf{r}_{12}\right)$ in Fig. 1(b). Moreover, $\Delta g^{\text {driver }}\left(\mathbf{r}_{12}\right)$ and $\Delta g^{\mathrm{PPL}}\left(\mathbf{r}_{12}\right)$ are largest in magnitude for short interelectronic distances, illustrating that the increasing basis set is required to capture short-ranged electronic correlation effects. Note that other than $g^{\text {driver }}\left(\mathbf{r}_{12}\right)$ and $g^{\mathrm{PPL}}\left(\mathbf{r}_{12}\right)$, the ring diagrams converge rapidly with respect to the employed number of orbitals. Likewise, we find that $g^{\text {rest }}\left(\mathbf{r}_{12}\right)$ converges significantly faster than $g^{\text {driver }}\left(\mathbf{r}_{12}\right)$ and $g^{\mathrm{PPL}}\left(\mathbf{r}_{12}\right)$, and we will not perform a more detailed analysis of this term in the present work.

Based on the decomposition of the electron pair correlation function introduced above, we now discuss the corresponding correlation energy contributions and their convergence to the complete basis set limit. To this end we have estimated the CBS limit reference energies by employing 1863 plane wave orbitals, which provides sufficiently well converged values for the purpose of the present discussion. We study an identical simulation cell to the one described above with a density corresponding to $r_{s}=5 a_{0}$. In addition, we discuss results obtained for $r_{s}=1.8 a_{0}$. Figures 1(c) and 1(d) show the convergence of the basis set incompleteness errors for the decomposed correlation energy retrieved as a function of the employed orbital basis set size ( $\Delta E^{\text {driver }}, \Delta E^{\text {ring }}, \Delta E^{\mathrm{PPL}}$, and $\left.\Delta E^{\text {rest }}\right)$. A comparison between Figs. 1(c) and 1(d) reveals that the convergence behavior of most terms is similar for both densities. Only $\Delta E^{\text {rest }}$ decays to zero in one case from above and in the other case from below. We find that the slowest decay of the basis set incompleteness error is observed for $\Delta E^{\text {driver }}$ and $\Delta E^{\mathrm{PPL}}$, in agreement with the discussion of the convergence of the corresponding pair correlation functions carried out in the paragraph above. $\Delta E^{\text {ring }}$ and $\Delta E^{\text {rest }}$ contributions exhibit a rapid convergence.

To better understand the reason for the convergence behavior of the ring and particle-particle ladder correlation energy contributions with respect to the employed basis, we perform an analysis of a simplified system. To this end we consider two electrons in a box with a homogeneous background charge such that $\mathbf{k}_{i}=\mathbf{k}_{j}=\mathbf{0}, \mathbf{k}_{a}=-\mathbf{k}_{b}=\mathbf{q}$ due to momentum conservation and the Coulomb integrals are $v_{i j}^{a b}=4 \pi / \mathbf{q}^{2}$. We separate the kinetic and the exchange part $\left(\delta_{q}\right)$ of one-electron energy differences $\Delta_{a b}^{i j}=-\left(\mathbf{q}^{2}+\delta_{q}^{2}\right)$, where the latter is negligible compared to the kinetic term for large magnitudes of $\mathbf{q}$. For the amplitudes $t_{i j}^{a b}$ we use a first-order approximation that reads $t_{i j}^{a b} \approx-4 \pi /\left(\mathbf{q}^{2}+\gamma^{2}\right)^{2}$, which corresponds to the Fourier transform of the Slater-type correlation function $-\gamma^{-1} e^{-\gamma\left|\mathbf{r}_{12}\right|}$. Replacing $t_{i j}^{a b}$ in the expressions for $S^{\text {ring }}(\mathbf{q})$ and $S^{\mathrm{PPL}}(\mathbf{q})$ by the above approximation in the limit of a large simulation cell yields

$$
S^{\mathrm{ring}}(\mathbf{q}) \approx \frac{1}{\mathbf{q}^{2}+\delta_{q}^{2}}\left(\frac{4 \pi}{\mathbf{q}^{2}} \frac{4 \pi}{\left(\mathbf{q}^{2}+\gamma^{2}\right)^{2}}\right)
$$

and

$S^{\mathrm{PPL}}(\mathbf{q}) \approx \frac{1}{\mathbf{q}^{2}+\delta_{q}^{2}}\left(\int d \mathbf{q}^{\prime} \frac{4 \pi}{\left(\mathbf{q}^{\prime}-\mathbf{q}\right)^{2}} \frac{4 \pi}{\left(\mathbf{q}^{\prime 2}+\gamma^{2}\right)^{2}}\right)$.

Note the duality relation of the terms in brackets: one is a pointwise product, the other a convolution of the Coulomb potential with the Slater-type correlation 
function. The latter turns into a pointwise product in real space $\gamma^{-1} e^{-\gamma\left|\mathbf{r}_{12}\right|} /\left|\mathbf{r}_{12}\right|$, which is the Yukawa potential scaled by $\gamma^{-1}$ whose Fourier transform is $\gamma^{-1} 4 \pi /\left(\mathbf{q}^{2}+\gamma^{2}\right)$. The important consequences of the above analysis are as follows: (i) the asymptotic behavior of the ring transition structure factor is $\lim _{|\mathbf{q}| \rightarrow \infty} S^{\text {ring }}(\mathbf{q}) \propto 1 / \mathbf{q}^{8}$, while (ii) the PPL transition structure factor decays as $\lim _{|\mathbf{q}| \rightarrow \infty} S^{\mathrm{PPL}}(\mathbf{q}) \propto 1 / \mathbf{q}^{4}$ only, which is identical to $\lim _{|\mathbf{q}| \rightarrow \infty} S^{\text {driver }}(\mathbf{q}) \propto 1 / \mathbf{q}^{4}$. This explains qualitatively the observed correlation energy convergence in Fig. 1 for the ring and PPL term with respect to the employed plane wave basis. Moreover, the above findings indicate that the PPL contribution becomes more important with decreasing electron density because $E^{\mathrm{PPL}} \propto \gamma^{-1}$ and $\gamma \propto 1 / r_{s}$ [25]. The above analysis also shows that the PPL contribution modifies the first-order coefficient in the Taylor expansion of $g\left(\left|\mathbf{r}_{12}\right|\right)$ with respect to $\left|\mathbf{r}_{12}\right|$ around $\left|\mathbf{r}_{12}\right|=0$ from above. It is important to note that although the previous analysis has been carried out for the UEG, its conclusions also apply to real (inhomogeneous) materials. This follows from the fact that the many-electron Hamiltonian is always dominated by the divergent electron-electron Coulomb repulsion at short interelectronic distances. Therefore, the asymptotics of Eqs. (5) and (6) as well as the electronic cusp conditions $[24,26]$ are universal. We note in passing that our findings for the asymptotic convergence of the PPL contribution agree with results obtained using a partial wave expansion in third-order perturbation theory outlined in Ref. [27].

Based on the analysis of the PPL term discussed above, we propose a finite basis set correction method for coupled cluster theory. Coupled cluster methods are becoming increasingly popular to perform $a b$ initio studies of solids and surfaces with high accuracy [28-35], demonstrating significant potential to further expand the scope of electronic structure theory calculations in, for example, the field of surface chemistry or the study of thermodynamic stabilities of solids. However, the underlying computational cost is much larger than that of the more efficient yet less accurate approximate density functional theory calculations. The main source of the computational cost in coupled cluster singles and doubles calculations originates from the PPL term. Its computational complexity scales as $\mathcal{O}\left(N_{v}^{4} N_{o}^{2}\right)$ in a canonical formulation, where $N_{v}$ and $N_{o}$ are the number of virtual and occupied orbitals, respectively. However, Fig. 1 and the discussion above show that the basis set incompleteness errors of the second-order correlation and the PPL term are proportional to each other albeit with an opposite sign. This observation motivates the following approximation to the CCSD correlation energy in the CBS limit that aims at accounting for the basis set incompleteness error in the driver $\left(E^{\text {driver }}\right)$ and PPL $\left(E^{\mathrm{PPL}}\right)$ term explicitly:

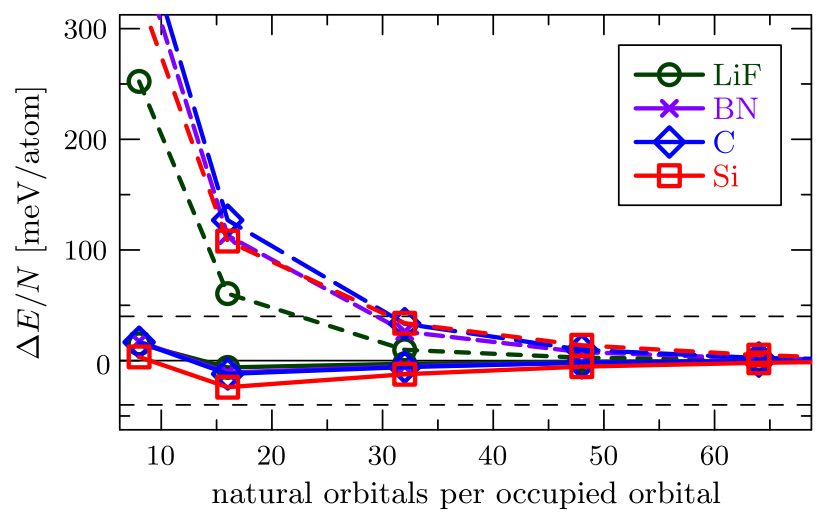

FIG. 2. CBS limit errors of CCSD (dashed) and CCSD PPL (solid) correlation energies for $a b$ initio systems with respect to the number of natural orbitals employing eight atomic supercells.

$E_{c}^{\mathrm{CCSD}-\mathrm{PPL}}(N)=E_{c}^{\mathrm{CCSD}}(N)+\Delta E^{\text {driver }}(N)+\Delta E^{\mathrm{PPL}}(N)$,

where

$$
\begin{gathered}
\Delta E^{\text {driver }}(N)=E^{\text {driver }}(\text { CBS })-E^{\text {driver }}(N), \\
\Delta E^{\mathrm{PPL}}(N)=\frac{\Delta E^{\text {driver }}(N)}{E^{\text {driver }}(N)} E^{\mathrm{PPL}}(N) .
\end{gathered}
$$

We refer to the employed orbital basis set size by $N$. $E^{\text {driver }}(\mathrm{CBS})$ can in practice be estimated in a computationally efficient manner using basis set extrapolation or explicit correlation techniques [25,36,37].

We now assess the efficiency of the proposed finite basis set correction to the coupled cluster correlation energy for $a b$ initio solid-state systems. All periodic calculations have been performed using the Vienna ab initio simulation package (VASP) [38] in the framework of the projector augmented wave method [39], interfaced to our coupled cluster code [40] that employs an automated tensor contraction framework [41]. We use natural orbitals to achieve a compact approximation for the virtual orbital space [42]. Figure 2 depicts the coupled cluster singles and doubles correlation energy convergence for a range of periodic crystals: $\mathrm{C}$ (diamond), $\mathrm{BN}$ (wurtzite), $\mathrm{LiF}$ (rocksalt), and $\mathrm{Si}$ (diamond), calculated with and without the proposed finite basis set correction. Our findings unequivocally demonstrate that the basis set convergence of the coupled cluster singles and doubles correlation energy is significantly faster including the proposed correction compared to uncorrected CCSD energies. Already 10 natural orbitals per occupied orbital agree with the CBS limit results to within chemical accuracy $(\approx 40 \mathrm{meV} /$ atom $)$ for the total energy per atom, as indicated by the region between the dashed horizontal lines. 80 natural orbitals per occupied orbital serve as the approximate CBS reference in the present work. In contrast to CCSD PPL, CCSD requires 
approximately 30 natural orbitals per occupied orbital to achieve a similar level of precision. We stress that a reduction in the number of virtual orbitals by a factor of 3 reduces the computational cost for the PPL term by 2 orders of magnitude.

We now seek to delineate the general purpose and scope of the proposed CCSD PPL method. Thus far, the carried-out analysis focused solely on total energies. However, many material properties depend on energy differences. We emphasize that the presented scheme is generally applicable but will only be beneficial for properties that exhibit a slow convergence with respect to the basis set size. In the case of chemical reaction energies that are dominated by weak van der Waals interactions only, the CCSD PPL scheme is not expected to achieve essential improvements. Furthermore, the CCSD PPL scheme will only be reliable if sufficiently large basis sets are employed, such that all terms other than PPL and driver are well converged (see Fig. 1). A more extensive investigation of atomic and molecular systems can be found in Ref. [43], which demonstrates that CCSD PPL atomization energies, reaction energies, ionization potentials, and electron affinities converge significantly faster with respect to the basis set size than conventional CCSD. This shows quantitatively that the conclusions drawn from the uniform electron gas hold for solids, molecules, and atoms for a wide range of properties.

Concluding remarks.-We have presented a diagrammatic decomposition of the coupled cluster pair correlation function and performed an analysis of the ring and particleparticle ladder terms. Based on their dual structure and the observed convergence with respect to the employed basis, we have introduced an efficient finite basis set correction that allows for reducing the computational cost of coupled cluster theory calculations in atoms, molecules, and solids substantially. In combination with the recently proposed finite size corrections [14] and other techniques $[25,40,44,45]$, this paves the way for a routine use of coupled cluster theory in electronic structure calculations of solids and surfaces. However, the significance of the present work extends beyond the proposed basis set correction. Given exact reference results for the spinresolved pair correlation function in ab initio or model systems that can be obtained using, for example, full configuration interaction quantum Monte Carlo calculations [35], the errors in the diagrammatic channels of approximate many-electron theories can be analyzed in detail, allowing for developing more accurate and better balanced truncations to the many-body perturbation expansion of the exact electronic correlation energy. Finally, we note that the introduced decomposition scheme can also help in developing more efficient embedding and local correlation theories, where the decoupling and decay of interelectronic correlation effects in different diagrammatic channels plays a crucial role and determines the accuracy of the employed approximations.
The authors thankfully acknowledge support and funding from the European Research Council (ERC) under the European Union's Horizon 2020 research and innovation program (Grant Agreement No. 715594). A. G. gratefully acknowledges many helpful discussions on F12 theory with Seiichiro Ten-no and David Tew. The computational results presented have been achieved in part using the Vienna Scientific Cluster (VSC).

andreas.grueneis@tuwien.ac.at

[1] W. Macke, Z. Naturforschr 5A, 192 (1950).

[2] D. Pines and D. Bohm, Phys. Rev. 85, 338 (1952).

[3] M. Gell-Mann and K. A. Brueckner, Phys. Rev. 106, 364 (1957).

[4] D. L. Freeman, J. Phys. C 16, 711 (1983).

[5] H. Yasuhara, Solid State Commun. 11, 1481 (1972).

[6] H. Yasuhara, J. Phys. Soc. Jpn. 36, 361 (1974).

[7] F. Coester and H. Kümmel, Nucl. Phys. 17, 477 (1960).

[8] J. Č́žzek, in Advances in Chemical Physics, edited by R. LeFebvre and C. Moser (John Wiley \& Sons, Inc., New York, 1969), pp. 35-89.

[9] T. M. Henderson and G. E. Scuseria, Mol. Phys. 108, 2511 (2010).

[10] J. Harl and G. Kresse, Phys. Rev. Lett. 103, 056401 (2009).

[11] J. F. Dobson, A. White, and A. Rubio, Phys. Rev. Lett. 96, 073201 (2006).

[12] D. Kats and F. R. Manby, J. Chem. Phys. 139, 021102 (2013)

[13] K. Liao and A. Grüneis, J. Chem. Phys. 145, 141102 (2016).

[14] T. Gruber, K. Liao, T. Tsatsoulis, F. Hummel, and A. Grüneis, Phys. Rev. X 8, 021043 (2018).

[15] C. Møller and M. S. Plesset, Phys. Rev. 46, 618 (1934).

[16] A. Szabo and N. S. Ostlund, Modern Quantum Chemistry: Introduction to Advanced Electronic Structure Theory (Dover Publications, Mineola, NY, 1996).

[17] I. Shavitt and R. J. Bartlett, Many-Body Methods in Chemistry and Physics: MBPT and Coupled-Cluster Theory (Cambridge University Press, Cambridge, England, 2009).

[18] J. J. Shepherd, T. M. Henderson, and G. E. Scuseria, J. Chem. Phys. 140, 124102 (2014).

[19] J. J. Shepherd, T. M. Henderson, and G. E. Scuseria, Phys. Rev. Lett. 112, 133002 (2014).

[20] R. F. Bishop and K. H. Lührmann, Phys. Rev. B 17, 3757 (1978).

[21] R. F. Bishop and K. H. Lührmann, Phys. Rev. B 26, 5523 (1982).

[22] R. F. Bishop, Theor. Chim. Acta 80, 95 (1991).

[23] A. Grüneis, J. J. Shepherd, A. Alavi, D. P. Tew, and G. H. Booth, J. Chem. Phys. 139, 084112 (2013).

[24] J. C. Kimball, Phys. Rev. A 7, 1648 (1973).

[25] A. Grüneis, Phys. Rev. Lett. 115, 066402 (2015).

[26] J. D. Morgan and W. Kutzelnigg, J. Phys. Chem. 97, 2425 (1993).

[27] W. Kutzelnigg and W. Klopper, J. Chem. Phys. 94, 1985 (1991).

[28] T. Gruber and A. Grüneis, Phys. Rev. B 98, 134108 (2018). 
[29] T. Tsatsoulis, S. Sakong, A. Groß, and A. Grüneis, J. Chem. Phys. 149, 244105 (2018).

[30] T. Tsatsoulis, F. Hummel, D. Usvyat, M. Schütz, G. H. Booth, S. S. Binnie, M. J. Gillan, D. Alfè, A. Michaelides, and A. Grüneis, J. Chem. Phys. 146, 204108 (2017).

[31] J. McClain, Q. Sun, G. K.-L. Chan, and T. C. Berkelbach, J. Chem. Theory Comput. 13, 1209 (2017).

[32] A. Kubas, D. Berger, H. Oberhofer, D. Maganas, K. Reuter, and F. Neese, J. Phys. Chem. Lett. 7, 4207 (2016).

[33] A. D. Boese and J. Sauer, J. Comput. Chem. 37, 2374 (2016).

[34] J. Yang, W. Hu, D. Usvyat, D. Matthews, M. Schütz, and G. K.-L. Chan, Science 345, 640 (2014).

[35] G. H. Booth, A. Grüneis, G. Kresse, and A. Alavi, Nature (London) 493, 365 (2013).

[36] J. J. Shepherd, A. Grüneis, G. H. Booth, G. Kresse, and A. Alavi, Phys. Rev. B 86, 035111 (2012).
[37] T. Schäfer, B. Ramberger, and G. Kresse, J. Chem. Phys. 146, 104101 (2017).

[38] G. Kresse and J. Furthmüller, Phys. Rev. B 54, 11169 (1996).

[39] P. E. Blöchl, Phys. Rev. B 50, 17953 (1994).

[40] F. Hummel, T. Tsatsoulis, and A. Grüneis, J. Chem. Phys. 146, 124105 (2017).

[41] E. Solomonik, D. Matthews, J. R. Hammond, J. F. Stanton, and J. Demmel, J. Parallel Distrib. Comput. 74, 3176 (2014).

[42] A. Grüneis, G. H. Booth, M. Marsman, J. Spencer, A. Alavi, and G. Kresse, J. Chem. Theory Comput. 7, 2780 (2011).

[43] A. Irmler and A. Grüneis, arXiv:1903.05559.

[44] G. H. Booth, T. Tsatsoulis, G. K.-L. Chan, and A. Grüneis, J. Chem. Phys. 145, 084111 (2016).

[45] T. N. Mihm, A. R. McIsaac, and J. J. Shepherd, J. Chem. Phys. 150, 191101 (2019). 